\title{
A hierarchical autonomous system based space information network architecture and topology control
}

\author{
ZHANG Wei ${ }^{1,2}$, ZHANG Gengxin $^{1}$, XIE Zhidong ${ }^{1}$, BIAN Dongming ${ }^{1}$, LI Yongqiang ${ }^{1}$ \\ 1 College of Communication Engineering, PLA University of Science and Technology, Nanjing 210007, China \\ 2 Department of Optical and Electrical Equipment, Equipment Academy, Beijing 101416, China
}

\begin{abstract}
SIN (Space Information Network) has recently emerged as a promising approach to solving the collaboration difficulty among current space programs. However, because of the SIN's large scale, high component complexity, and dynamic characteristics, designing a proper SIN architecture is challenging. Firstly, we propose a novel SIN architecture, which is composed of GEO (Geostationary Earth Orbit) satellites as backbone network nodes, LEO (Low Earth Orbit) or other types of satellites as enhanced coverage nodes, and high-altitude platforms to meet the service requirements of emergency or hotspot applications. Unlike most existing studies, the proposed architecture is AS (Autonomous-System) based. We decouple the complex SIN into simpler sub-networks using a hierarchical AS model. Then, we propose a topology control algorithm to minimize the time delay among sub-AS networks. We prove that the proposed algorithm achieves logical $k$-connectivity provided that the original physical topology has $k$-connectivity. Simulation results validate the theoretic analysis and effectiveness of the algorithm.
\end{abstract}

Key words: space information network, architecture, autonomous system, topology control, time delay, strong connectivity

Citation: ZHANG W, ZHAGN G X, XIE Z D, et al. A hierarchical autonomous system based space information network architecture and topology control[J]. Journal of communications and information networks, 2016,1(3): 77-89.

\section{Introduction}

Space information and networking research have added a new scientific and engineering era to spaceexploration history. In the early phase, ground stations shot radio signals toward satellite antennas whenever they came into view; the system's coverage and service ability was limited. Currently, among the various kinds of space programs, most systems are single layered, such as Inmarsat, O3b, GPS and Iridium ${ }^{[1-4]}$. These systems provide particular information services using their own strengths, and the designs have always been targeted to meet the services. However, in many applications, an integrated service is needed in addition to a specialized service. For instance, ATC (Air-Traffic Control) needs integrated communication, navigation, and remote sensing services ${ }^{[5]}$. However, current space systems

Manuscript received May 4, 2016; accepted Jul. 16, 2016

The work is supported by the National Natural Science Foundation of China (Nos.91338201, 91438109, 61401507). 
are not universal and differ from one mission to another. This phenomenon in turn led individual space programs to operate their own specialized space-information networks. The immediate answer is to develop a space-information network that can be standardized, interconnected, and easily evolved over future decades. Such motivations led to the development of networking architectures and model research for space-information networks.

SIN is a new type of self-organizing system composed of space, air, land, and sea information systems ${ }^{[6-10]}$. It can integrate various types of platforms as network nodes, e.g., satellites, HAPs (High-Altitude Platform stations), aerial vehicles, and sensors. To meet the various service requirements, SIN nodes can be distributed to different altitudes, work in different environments, and have different computing or storage abilities. Thus, the SIN nodes' behaviors are complicated, and the network structure is threedimensional and multi-layered. Moreover, satellites, HAPs, and aerial vehicles move in and out of sight, so the SIN topology is dynamic. Because of these distinguishing characteristics, SIN's architecture design and topology control is challenging.

Existing studies on SIN architecture mainly focus on ML-SNs (Multilayered Satellite Networks). Three main layers can be categorized, based on the orbital distance from the Earth: GEO layer, LEO layer, and MEO (Medium Earth-Orbit) layer. MLSNs usually take two forms ${ }^{[11-15]}$, double-layered satellite networks based on MEO/LEO or GEO/LEO satellites, and three-layered satellite networks based on GEO/MEO/ LEO satellites. Since Kimura first proposed a doublelayer satellite constellation made of MEO/LEO satellites in the $1990 \mathrm{~s}^{[16]}$, MLSNs have attracted many researchers. J. Lee and S. Kang proposed a new multilayered and hierarchical satellite network architecture called SoS (Satellite over Satellite), and designed a corresponding routing protocol for hierarchical satellite networks called HSRP (Hierarchical Satellite
Routing Protocol) ${ }^{[17]}$. Ian F. Akyildiz et.al proposed an IP-based routing algorithm for multilayered satellite networks called MLSR (Multi-Layered Satellite Routing $)^{[18]}$. The algorithm is also suitable for threelayered satellite networks consisting of GEO/MEO/ LEO. However, in most studies, all the MLSN layers overlap and have similar functions. MLSN links are complicated and inconvenient to maintain.

In this article, we first propose a novel SIN architecture, which is composed of GEO satellites as backbone network nodes, LEO or other satellite types as enhanced coverage nodes, and high-altitude platforms to meet the service requirements of emergency or hot-spot applications. Compared with most existing studies, the proposed architecture is AS (AutonomousSystem) based. An AS network is a collection of nodes with similar properties. The reasons for using an AS approach are twofold. Firstly, the complex SIN is decoupled into a series of AS networks. Each AS network can have an independent control and transmission strategy. Secondly, the dynamic AS network can be divided into small semi-static sub-AS networks to simplify the control of each AS. Then, we propose a topology control algorithm to minimize the time delay among the AS networks. The proposed algorithm preserves the $k$-connectivity of the original network, and is min-max delay optimal. A min-max algorithm tries to minimize the maximumend-to-end delay between any pair of nodes in the network.

The rest of this article is organized as follows. In Section 2, the proposed SIN architecture is described. In Section 3, we define the AS network model and decouple the SIN into AS networks. In Section 4, we propose a topology control algorithm to achieve low time delay and strong connectivity between sub-AS networks. Then, the validity of the proposed algorithm is proved in Section 5. In Section 6 , simulation results are presented and discussed. Finally, we conclude with Section 7. 


\section{SIN architecture}

In this section, we present the SIN architecture. As stated above, SIN is a new type of self-organizing system comprised of heterogeneous platforms to meet various information-service requirements. However, if we simply overlap the different space programs as MLSNs, the SIN topology will be too complex, and not realistic in practice. Thus, in the proposed architecture, we use a GEO backbone as the foundation of the SIN, and the other parts of the SIN to enhance or access units. As demonstrated in Fig.1, the SIN architecture is composed of five main parts: the GEO backbone network, service-enhanced satellite network, high-altitude platform network, operation and control network, and access network.

(1) GEO backbone network. The GEO backbone consists of three to five GEO positions connected as a loop by high-speed laser links. Each GEO position may contain several GEO satellites cooperating as a DSC (Distributed Satellite Cluster). The DSC is an alternative to a monolithic satellite with advantages of stronger functions, lower risk, easy evolution, etc. ${ }^{[19,20]}$. A DSC can be physically composed of communication, navigation, relay, or remote-sensing satellites; however, we use a DSC as a logical node in the topology. The inherent character of GEO satellites (e.g., stationary over the Earth, vast coverage, and mature technology) make them especially suitable for being backbone nodes. As shown in Fig.2, three GEO satellites (positions) can cover 92.15\% of the Earth's surface with full coverage time.

(2) Service-enhanced satellite network. The service-enhanced satellite network supplements the GEO backbone and improves the SIN coverage performance in the mid-high latitudes and polar regions. This service-enhanced network is optional (illustrated with dotted lines in Fig.1) in the early stages of the SIN, and can be gradually extended. For instance, as demonstrated in Fig.3, only nine LEO satellites, evenly distributed in one polar orbit at an altitude of $1450 \mathrm{~km}$, can cover the polar area well.

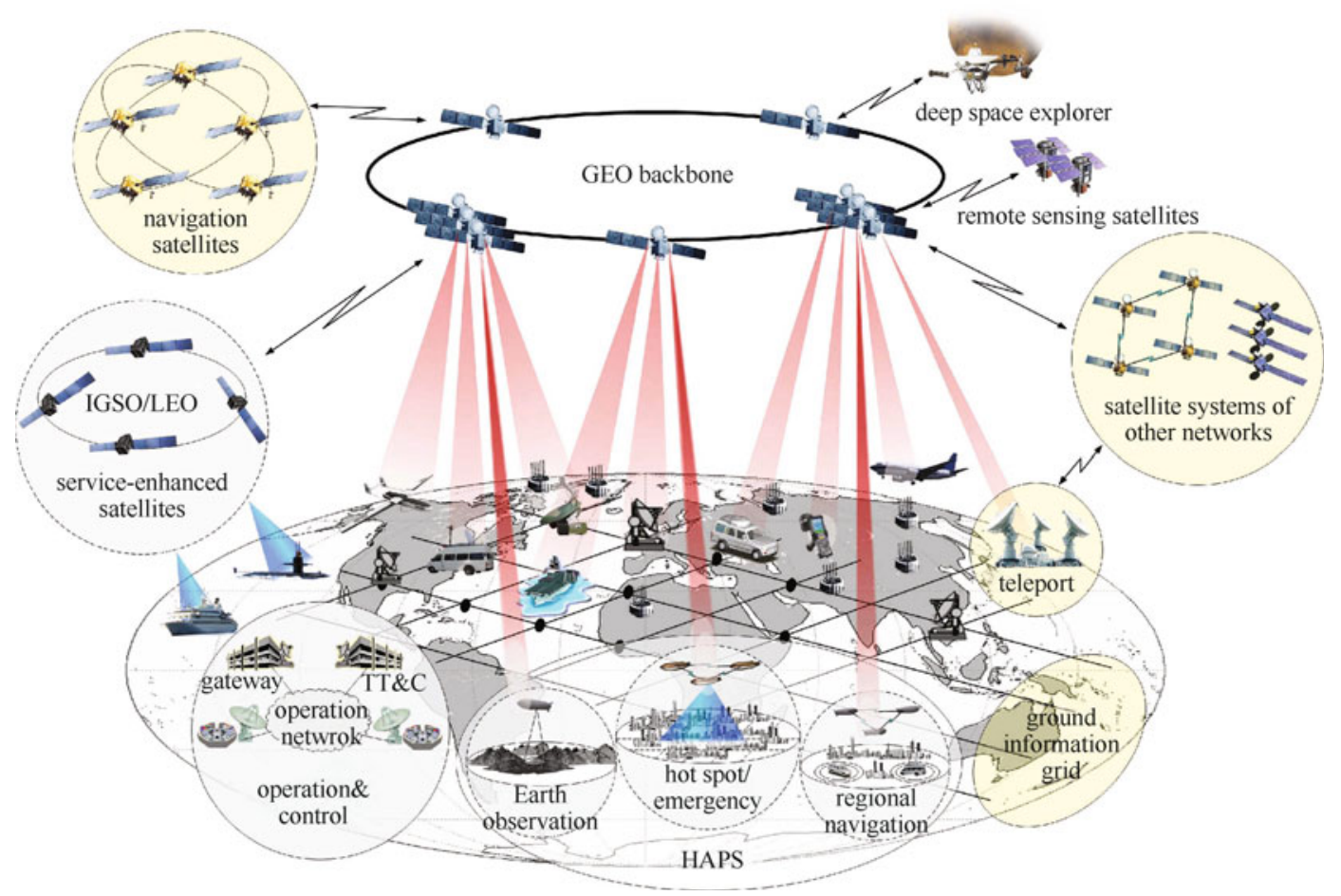

Figure 1 SIN architecture 


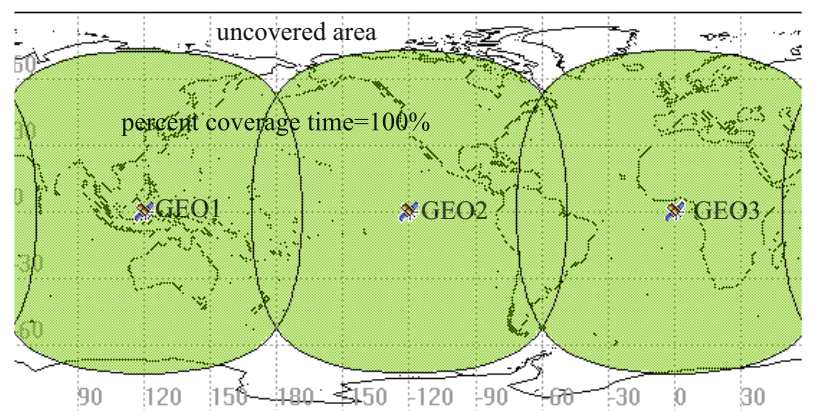

Figure 2 Coverage time of 3 GEO satellites (positions) in STK. The minimum ground elevation angle is 10 degree. The green area is $100 \%$ covered by GEO satellites, and the white area is uncovered

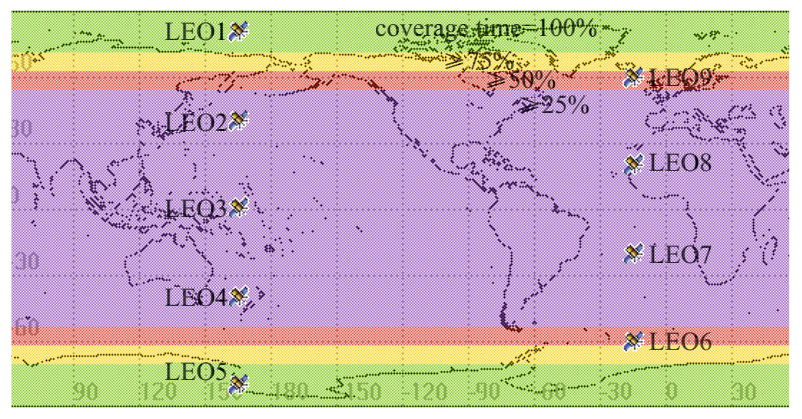

Figure 3 Coverage time of 9 LEO satellites in STK

These nine LEO satellites are connected as a loop by ISLs (Inter-Satellite Links). They relay the backbone information to the uncovered GEO area. Although the SIN contains different satellite orbits, it is based on a GEO backbone. Unlike MLSNs, other satellite orbits only play enhanced roles to provide better coverage with lower cost.

(3) High altitude platform network. Considering the limited satellite resources, e.g., orbit, frequency, and launching cost, services that only rely on satellite platforms are costly, especially in hotspot areas. However, because of the distinguishing characteristics of HAPs, e.g., low cost, low time delay, and high server capacity ${ }^{[21,22]}$, they can make up for the insufficient service of satellite platforms in city areas. With their quick reaction capability, highaltitude platforms can be rapidly deployed to meet the demands for emergency rescue. Moreover, they can be used for regional navigation in areas where satellite signals are shadowed. They can also be used as Earth observers in some situations. HAPs in the network exchange information using high-speed laser or microwave links.

(4) Operation and control network. The main tasks of the operation and control network are to maintain the normal operation of various types of platform, and to provide operational support for users' service needs. The network is composed of a network control subsystem, application management subsystem, TT\&C (Telemetry Track and Command) stations, and gateway stations.

(5) Access network. The SIN access network mainly refers to ground users, low-altitude terminals, ground information grid networks, navigation satellites, Earth observation satellites, deep-space explorers, and satellite systems of other networks. These components usually exist before SIN emerges, and are gradually integrated into it. We color them yellow in Fig.1.

1) Ground and low-altitude terminals include sensors, hand-held devices, portable terminals, fixed stations, vehicle terminals, ship-based terminals, and unmanned/manned aerial vehicles. These terminals can access the SIN through the GEO backbone network, service-enhanced satellite network, or highaltitude platform network.

2) Low-orbit spacecraft include various types of Earth-observation satellites, missiles, and rockets. The coverage area of a low-orbit spacecraft is small, and the travel speed is high. It is difficult to maintain a stable link between these spacecraft and ground stations. However, using the GEO backbone as a relay, the information from a low-orbit spacecraft to ground is continuous.

3) Navigation satellites access the SIN through the GEO backbone network, which transmits the navigation data to other sub-networks or users to enhance the navigation services. 
4) Deep-space explorers. With the GEO backbone as a relay, communication between a deep-space explorer and an Earth station can take two steps. Firstly, the data can travel from the explorer to the GEO satellite, unaffected by atmospheric conditions; secondly, the data continues from the GEO satellite to the Earth station using mature technologies.

5) Satellite systems of other networks. These systems usually exist before the SIN emerges. They can either access the SIN through a teleport station or through ISLs with the GEO backbone. The function of a teleport station is similar to that of a gateway station; it connects the GEO backbone network, ground network, and other satellite systems.

6) Ground network. The ground information grid network in Fig. 1 is the ground backbone network. It accesses the SIN through gateway stations. Thus, we have a double backbone network in space and on the ground in the proposed architecture.

\section{Network model}

In this section, the AS network model is defined.
As demonstrated in Fig.1, SIN is a new type of selforganizing system composed of heterogeneous platforms to meet various information-service requirements. SIN nodes are distributed at different altitudes and work in different environments in either mobile (e.g., in HAP networks) or static (e.g., in terrestrial wireless sensor networks) conditions. As demonstrated in Fig.4, the SIN topology is complex. If we apply a unified strategy to manage the entire SIN, it will induce low efficiency, and fail to maintain normal network operation with too much control information. Thus, as shown in Fig.5, we divide the SIN into a series of AS networks according to the nodes' properties. Each AS network can adopt an independent topology-control strategy. AS-1 contains all the SIN satellites and its topology is usually highly dynamic but predictable. AS-2 contains the HAPs, AS- 3 contains the airplanes, and AS- 4 contains the ground nodes. The properties of AS-2/3/4 are similar: their nodes move slowly and connect to each other autonomously.

As shown in Fig.6, each AS network can be further separated into sub-AS networks. With this

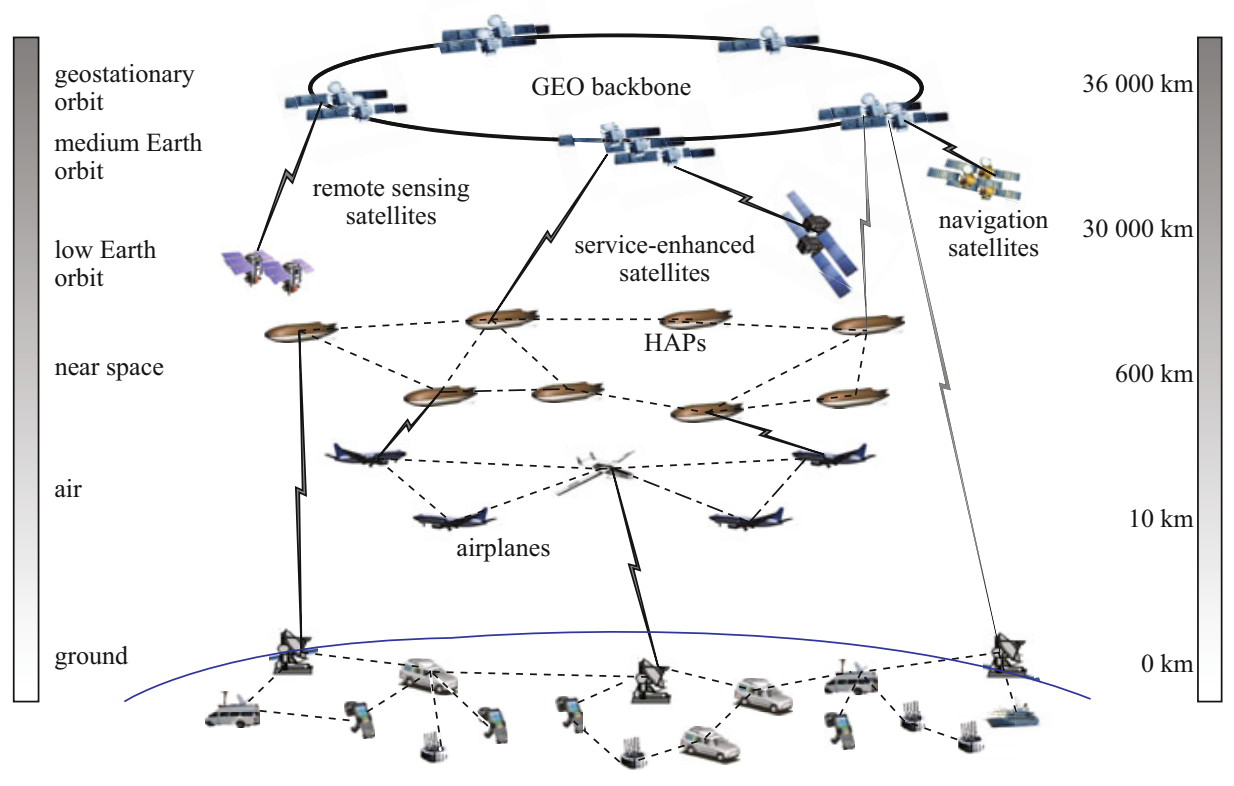

Figure 4 Topology of SIN is complex 


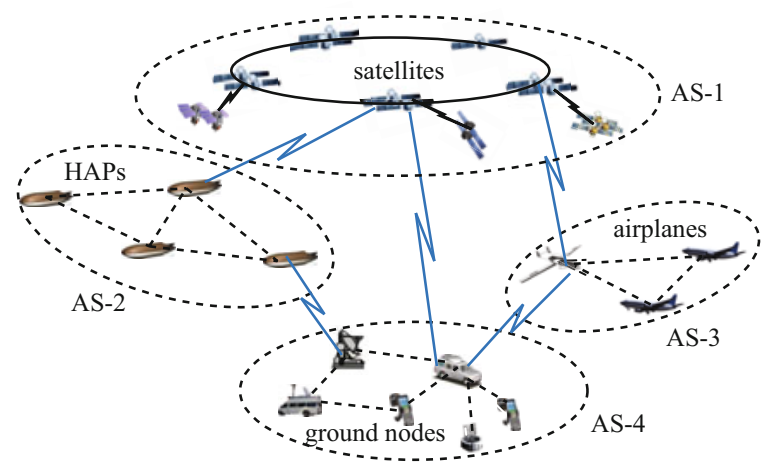

Figure 5 The whole SIN is divided into a series of AS networks according to the property of the nodes

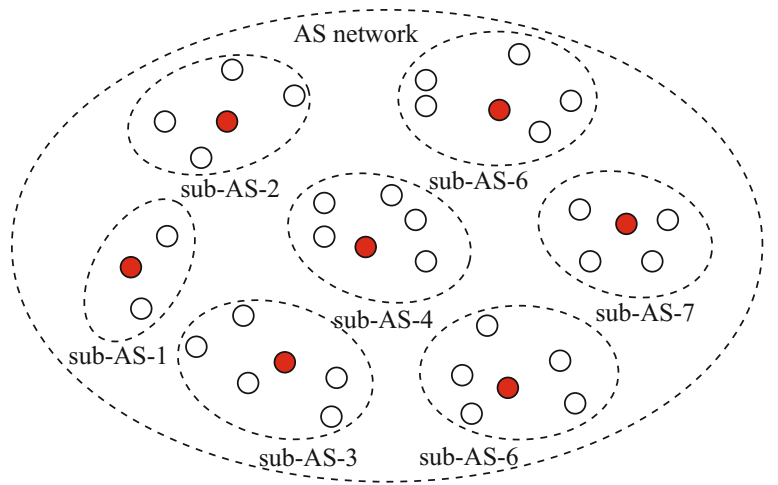

Figure 6 Nodes in the AS network autonomously form sub-AS networks and elect sub-AS cores

hierarchical AS model, the dynamic AS network is decoupled into semi-static local sub-AS networks. Thus, the SIN is easier to control. Nodes in the AS network autonomously form sub-AS networks and elect sub-AS cores. Each core dominates the topology control of the intra-sub-AS network and inter-sub AS network. That is, this node will be the member of the sub-AS dominated by its parent core. The AS-1 topology control is usually based on the satellite constellations' design; we will not discuss it in this article. The rest of this article mainly focuses on the AS-2/3/4 inter-sub-AS topology control method.

Considering that the properties of nodes in an AS network are similar, we assume that nodes in the same AS network are homogeneous and have the same maximal transmission range $R_{\max }$. Let an AS network topology be represented by an undirected simple graph $G=(V, E)$, where $V=\left\{u_{1}, u_{2}, \cdots, u_{n}\right\}$ is the set of nodes (or equivalently, vertices) and $E=$ $\left\{\left(u_{i}, u_{j}\right) \mid\left(u_{i}, u_{j} \in V\right) \wedge\left(r\left(u_{i}, u_{j}\right) \leqslant R_{\max }\right)\right\}$ is the set of links (edges). $r\left(u_{i}, u_{j}\right)$ is the distance between nodes $u_{i}$ and $u_{j}$. Each node is assigned a unique identifier (ID) according to its property, e.g., MAC address.

We assume that $G$ is a general graph, i.e., if $(u, v)$ $\in E, u$ and $v$ can exchange information with each other. We also assume that the link is symmetric and obstacle-free, and each node can obtain its location information by some means (e.g., GPS). We next define several graphs related terms, which will be used in both algorithms and proofs. For all definitions, we refer to graph $G=(V, E)$ and sub-graphs $G_{i}=\left(V_{i}\right.$, $\left.E_{i}\right)$ and $G_{j}=\left(V_{j}, E_{j}\right)$.

Definition 1 Weight function. For an edge $e=(u, v)$, the weight function is $w(u, v)=(d(u, v), \min (I D(u)$, $I D(v)), \max (I D(u), I D(v)))$, where $d(u, v)$ is the time delay between $u$ and $v$ when exchanging information. Given $\left(u_{1}, v_{1}\right),\left(u_{2}, v_{2}\right) \in E$, the relationship between $w\left(u_{1}, v_{1}\right)$ and $w\left(u_{2}, v_{2}\right)$ is given as

$$
\begin{aligned}
& w\left(u_{1}, v_{1}\right)>w\left(u_{2}, v_{2}\right) \Leftrightarrow d\left(u_{1}, v_{1}\right)>d\left(u_{2}, v_{2}\right) \\
& \text { or }\left(d\left(u_{1}, v_{1}\right)=d\left(u_{2}, v_{2}\right)\right) \wedge \\
& \left(\min \left(I D\left(u_{1}\right), I D\left(v_{1}\right)\right)>\min \left(I D\left(u_{2}\right), I D\left(v_{2}\right)\right)\right) \\
& \text { or }\left(d\left(u_{1}, v_{1}\right)=d\left(u_{2}, v_{2}\right)\right) \wedge \\
& \left(\min \left(I D\left(u_{1}\right), I D\left(v_{1}\right)\right)=\min \left(I D\left(u_{2}\right), I D\left(v_{2}\right)\right)\right) \wedge \\
& \left(\max \left(I D\left(u_{1}\right), I D\left(v_{1}\right)\right)>\max \left(I D\left(u_{2}\right), I D\left(v_{2}\right)\right)\right) .
\end{aligned}
$$

It is obvious that edges with the same vertices have equivalent weights. However, edges with different end-vertices have different weights.

Definition $2 k$-connected. In graph (topology) $G$, node $u$ is said to be connected to node $v$, if there exists a path $p=\overline{u x_{1} x_{2} \cdots x_{m 1} x_{m v}}$, where $x_{i} \in V$ and $\left(u, x_{1}\right),\left(x_{i}, x_{j}\right),\left(x_{m}, v\right) \in E$. Further, for any $u, v \in V$, if there exists at least $k$ disjoint paths between them. Graph $G$ is $k$-connected, and denoted by $C O N(G$, $k$ ). If $G$ is $k$-connected, it follows that, there does not exist a set of $k-1$ vertices, whose removal will partition $G$ into two or more connected components. 
Definition 3 Neighboring $k$-connected sub-graphs. For two disjoint sub-graphs $G_{i}$ and $G_{j}$ of $G$, if $\exists u \in$ $V_{i}, v \in V_{j}$ and $\exists(u, v) \in E, G_{i}$ and $G_{j}$ are neighboring sub-graphs, denoted by $N B R_{G}\left(G_{i}, G_{j}\right)$. If $\operatorname{CON}\left(G_{i}\right.$, k) $\wedge \operatorname{CON}\left(G_{j}, k\right)$, and $\exists\left(u_{1}, v_{1}\right), \cdots,\left(u_{k}, v_{k}\right) \in E$, where $u_{1}, \cdots, u_{k} \in V_{i}$ and $v_{1}, \cdots, v_{k} \in V_{j}, G_{i}$ and $G_{j}$ are neighboring $k$-connected sub-graphs, denoted by $N B R_{G}\left(G_{i}, G_{j}, k\right)$.

Definition 4 Multihop $k$-connected sub-graphs. Let $G_{1}, G_{2}, \cdots, G_{n}$ be a partitioning of $G$. If $\exists G_{l}$ subject

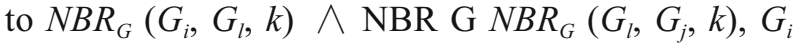
and $G_{j}$ are multihop $k$-connected sub-graphs, denoted $\operatorname{byMCON}_{G}\left(G_{i}, G_{j}, k\right)$.

\section{Algorithms for inter-sub-AS topology control}

In this section, we propose an inter-sub-AS topology control algorithm. Considering the scale of a SIN, a substantial proportion of the links are very long. Excessive use of these longdistance links not only brings additional delay but also reduces the system efficiency $^{[23,24]}$. Thus, in the SIN topology control, end-to-end delay should not be ignored. Instead of using long links in the proposed algorithm, nodes in different sub-ASs collaboratively determine which links should be used to form proper neighbor relations, and unnecessary links are removed. As a result, the network topology may be susceptible to unpredictable events, such as hardware failures. Therefore, to design a robust topology-control algorithm, the networks' $k$-connectivity is considered. The failure of less than $k^{-1}$ nodes will not disconnect the $k$-connectivity network.

As mentioned above, nodes in the AS network autonomously form sub-AS networks and elect sub-AS cores using the backbone-based algorithm proposed in Ref.[25]. In order to allow adjacent sub-AS networks to discover each other every node continue broadcasting hello message (NodeID,
Location, CoreID, Degree, Delay) periodically. The explanation of each field is as follows. 1) NodeID: the unique ID of each node. 2) Location: the location of each node. 3) CoreID: the ID of the core with which the sending node is currently associated. Note that a core node uses its own ID for this field. 4) Degree: the degree of connectivity (the number of neighboring nodes). 5) Delay: time delay to each neighboring node when exchange information. When a node $u$ receives a hello message from a node $v$ that belongs to a different sub-AS (e.g., they have different CoreID), $u$ will place $v$ 's information in its border list. Then this border list is reported to the node's parent core. With these border lists, we present a distributed algorithm for inter-sub-AS. This algorithm is described in Algorithm 1. Where $G$ represents the AS, and let $G_{1}$, $G_{2}, \cdots, G_{n}$ (sub-AS) be a partitioning of $G$.

In this algorithm, the core of a sub-AS $A$ checks whether there exist $k$ disjoint links from this subAS to each adjacent sub-AS $B$. That is accomplished by applying an algorithm (MaxMatching) ${ }^{[26]}$ that computes a matching of maximum cardinality in a bipartite graph defined by the nodes in respective subAS networks and the edges with one vertex in each sub-AS. If $k$ does not exceed the size of maximum cardinality matching, the core of sub-AS $A$ select $k$ disjoint links that meet the min-max delay optimal. When there does not exist $k$ disjoint links between $A$ and $B$ (only $k_{m}$ disjoint links), the core preserve the $k_{m}$-connectivity between these two sub-AS networks and minimize the maximum delay between them. Note that this connectivity preservation $\left(k_{m}{ }^{-}\right.$ connectivity) cannot guarantee $k$-connectivity between sub-AS $A$ and $B$. However, global $k$-connectivity can be guaranteed after Algorithm 1 is completed as long as the original physical topology is $k$-connectivity. This will be proved in Section 5.

Parameter $D_{I A}\left(G_{1}, G_{2}\right)$ in Algorithm 1 is used to perform an optimization which removes unnecessary links between certain adjacent sub-AS networks, 
while preserving the connectivity of the resulting topology. $D_{I A}\left(G_{1}, G_{2}\right)$ is the maximum delay of the selected $k$ links. However, when the number $k_{m}$ of disjoint links between two adjacent sub-AS networks is less than $k, D_{I A}\left(G_{1}, G_{2}\right)$ is $\infty$. Then, sub-AS $A$ will not connect to a neighboring sub-AS $B$ directly if it observes that there exists another sub-AS $C$, where $C$ is also a neighbor of $B$, and both $D_{I A}\left(G_{A}, G_{C}\right)$ and $D_{I A}$ $\left(G_{B}, G_{C}\right)$ are less than $D_{I A}\left(G_{A}, G_{B}\right)$.

After Algorithm 1 is completed, each border node is as-signed an inter-AS link list, and border nodes of different sub-AS connect each other according to these lists. This topology will be maintained by every node with hello message periodically, and always preserve the objective connectivity of the network.

Tab. 1 shows the average control messages utilized to complete the topology algorithm for each subAS. Let $N$ be the total number of nodes in the AS network. Let $S$ be the number of sub-AS networks, and $N_{S}$ be the average number of nodes per sub-AS, i.e., $N_{S}=N / S$. Let $R_{B}$ be the average probability of nodes that are border nodes in a sub-AS, where $0<$ $R_{B}<1$. Let $S_{N}$ be the average number of neighboring sub-AS networks for each sub-AS, i.e., $0<S_{N}<S$. We partition Algorithm 1 into major steps. Hence, from Tab.1, the total number of control messages required in the AS is $S\left(N_{S} R_{B}+2 S_{N}+2\right)$. It can be simplified as $R_{B} N+2 S_{N} S+2 S$, which is $o(N)+$ $o\left(S_{N} S\right)$ in the worst case.

Algorithm 1: Inter-sub-AS topology control

Input: (at sub-AS $G_{k}=\left(V_{k}, E_{k}\right)$ )

$k$ (required connectivity)

\section{Output:}

Links for all nodes in $G_{k}$ 's border list.

\section{Begin:}

$$
\begin{aligned}
& G_{k i}=\left(V_{k i}, E_{k i}\right), V_{k i} \leftarrow V_{k}, E_{k i} \leftarrow \varnothing \\
& \text { for all } G_{i} \text { subject to } \operatorname{NBR}_{G}\left(G_{k}, G_{i}\right) \text { do } \\
& \quad V^{\prime} \leftarrow\left\{v \mid\left(v \in G_{i}\right) \wedge\left(v \text { is adjacent to } G_{k}\right)\right\} \\
& \quad V_{k i} \leftarrow V_{k i} \cup V^{\prime}
\end{aligned}
$$

$$
\begin{aligned}
& E_{k i} \leftarrow\left\{(u, v) \mid \begin{array}{l}
\left(u \in V_{k}\right) \wedge\left(v \in V^{\prime}\right) \wedge \\
\left(r(u, v) \leqslant R_{\max }\right)
\end{array}\right\} \\
& M \leftarrow \varnothing \\
& E_{a} \leftarrow \text { sort all edges in } E_{k i} \text { in ascending order of }
\end{aligned}
$$
weight (as defined in Definition 1)

$k_{m} \leftarrow \mid$ MaxMatching $\left(G_{k i}\right) \mid$

$\% \mid$ MaxMatching $\left(G_{k i}\right) \mid$ is the number of edges in MaxMatching $\left(G_{k i}\right)$

$$
\text { if } k_{m} \geqslant k \text { then }
$$

for all edges $e_{t}=\left(u_{t}, v_{t}\right) \in E_{a}$ in the order

do

Find the smallest $t$, subject to $|M| \geqslant k$, where $M \leftarrow$ MaxMatching $\left(G_{t}=\left(V_{k i}, E_{a}(t)\right)\right)$, and $|M|$ is the number of edges in $M, E_{a}(t)=\left\{e_{1}, \cdots, e_{t}\right\}$

\section{end for}

$L\left(G_{k}, G_{i}\right) \leftarrow M$, where $\left|e_{t}\right|$ is the weight of $e_{t}$

$$
L\left(G_{k}, G_{i}\right) \leftarrow M
$$

else

for all edges $e_{t}=\left(u_{t}, v_{t}\right) \in E_{a}$ in the order do

Find the smallest $t$, subject to $|M| \geqslant k_{2}$, and $M \leftarrow$ MaxMatching $\left(G_{t}=\left(V_{k i}, E_{a}(t)\right)\right)$

\section{end for}

$$
\begin{aligned}
& D_{I A}\left(G_{k}, G_{i}\right) \leftarrow \infty \\
& L\left(G_{k}, G_{i}\right) \leftarrow M
\end{aligned}
$$

\section{end if}

Send $D_{I A}\left(G_{k}, G_{i}\right)$ to neighbor sub-AS

\section{end for}

Collect $D_{I A}$ from neighboring sub-AS

LIST $\leftarrow \varnothing$

for all $G_{p}$ subject to $N B R_{\mathrm{G}}\left(G_{k}, G_{p}\right)$ do

if there does not exist $G_{q}$ subject to

$$
\begin{aligned}
& N B R_{\mathrm{G}}\left(G_{k}, G_{q}\right) \wedge N B R_{\mathrm{G}}\left(G_{k}, G_{p}\right) \wedge \\
& \left(D_{I A}\left(G_{k}, G_{q}\right)<D_{I A}\left(G_{k}, G_{p}\right)\right) \wedge \\
& \left(D_{I A}\left(G_{p}, G_{q}\right)<D_{I A}\left(G_{k}, G_{p}\right)\right) \text { then } \\
& \quad L I S T \leftarrow L I S T \cup L\left(G_{k}, G_{i}\right)
\end{aligned}
$$

\section{end if}

end for 


\section{Proof of strong connectivity}

In this section, we prove that the strong connectivity of Algorithm $1^{[27]}$. The results are given as the following theorems. Note that, we have not present intra-sub-AS topology control algorithms in this article. However, different intra-sub-AS topology control algorithms may be executed in sub-AS networks before Algorithm 1 is executed. But in order to achieve global $k$-connectivity, nodes of any intrasub-AS should be $k$-connected. That is, for every subAS $G_{i}$, we know that $\operatorname{CON}\left(G_{i}, k\right)$ is true.

Theorem 1 Let $G=(V, E)$ be the initial topology of an AS. Let $G^{\prime}=\left(V, E^{\prime}\right)$ be the topology after Algorithm 1 is completed. Then we have $\operatorname{CON}(G, k)$ $\Leftrightarrow \operatorname{CON}\left(G^{\prime}, k\right)$.

Before proving the correctness of Theorem 1, several lemmas used in that proof are first provided.

Lemma 1 Let $G_{i}=\left(V_{i}, E_{i}\right)$ and $G_{j}=\left(V_{j}, E_{j}\right)$ be two sub-graphs of graph $G$. If $N B R_{G}\left(G_{i}, G_{j}, k\right)$, then $C O N$ $\left(G_{i} \cup_{G} G_{j}, k\right)$.

Proof of Lemma 1 In order to prove $\operatorname{CON}\left(G_{i} \cup_{G} G_{j}\right.$, $k$ ), we prove $G_{i} \cup_{G} G_{j}$ is connected with the removal of any $k-1$ vertices from it. Since $N B R_{G}\left(G_{i} G_{j}, k\right)$, we have $\operatorname{CON}\left(G_{i}, k\right)$ and $\operatorname{CON}\left(G_{j}, k\right)$, i.e., consider any $u$, $v \in G_{i}$ or $u, v \in G_{j}, u$ is $k$-connected to $v$. Then we only need to consider the case $\left(u \in G_{i}\right) \wedge\left(v \in G_{j}\right)$.

Since $N B R_{G}\left(G_{i} G_{j}, k\right), \exists u_{0} \in G_{i}, v_{0} \in G_{j}, u_{0}$ is connected to $v_{0}$ with the removal of any $k-1$ vertices from $V_{i} \cup V_{j}-\left\{u_{0}, v_{0}\right\}$. With $\operatorname{CON}\left(G_{i}, k\right)$ and $\operatorname{CON}\left(G_{j}, k\right)$, we know that $u$ is connected to $u_{0}$, and $v$ is connected to $v_{0}$. Hence $u$ is connected to $v$. That is, $G_{i} \cup_{G} G_{j}$ is connected with the removal of any $k-1$ vertices from it. Corollary 1 Let sub-graphs $G_{1}, G_{2}, \cdots, G_{n}$ be a partitioning of $G$. Let $S_{m}$ be the maximal set of subgraphs subject to $\forall G_{i}, G_{j} \in S_{m}, \exists \operatorname{MCON}_{G}\left(G_{i}, G_{j}, k\right)$. Then, $\cup_{G}\left\{G_{i} \mid G_{i} \in S_{m}\right\}$ is $k$-connected.

Lemma 2 Let $G_{s}$ be a sub-graph of $G$, and let $G_{s}{ }^{\prime}$ be edges reduction of $G_{s}$. Let $G^{\prime \prime}=\left(V, E^{\prime}\right)=\left(G-G_{s}\right) \cup_{G}$ $-G_{s}{ }^{\prime}$. If $\operatorname{CON}\left(G_{s}, k\right) \wedge \operatorname{CON}\left(G_{s}{ }^{\prime}, k\right) \wedge \operatorname{CON}(G, k)$, then $\operatorname{CON}\left(G^{\prime \prime}, k\right)$.

Proof of Lemma 2 In order to prove $\operatorname{CON}\left(G^{\prime \prime}, k\right)$, we prove $\forall u, v \in G^{\prime \prime}$ is connected with the removal of any $k^{-1}$ vertices from $G^{\prime \prime}$. Without loss of generality, three cases are considered in the following.

1) $u, v \in V_{s}$ : It is obvious true because of $\operatorname{CON}\left(G_{s}{ }^{\prime}, k\right)$.

2) $u \in V_{s}$ and $v \in V-V_{s}$ : Since $\operatorname{CON}(G, k), u$ is connected to $v$ in path $p$ with the removal of any $k-1$ vertices in $G$. If $p \subseteq E-E_{s}, p$ is also exist in $G^{\prime \prime}$, $u$ is connected to $v$ by removing those $k^{-1}$ vertices. Otherwise, $\exists(a \in p) \wedge\left(a \in V_{s}\right)$ and $a$ is connected to $v$ in $G-G_{s}$. Since $\operatorname{CON}\left(G_{s}{ }^{\prime}, k\right), u$ is connected to $a$ by removing those $k-1$ vertices. Then $u$ is connected to $v$ with the removal of any $k-1$ vertices in $G^{\prime \prime}$.

3) $u, v \in V-V_{s}$ : Similarly, since $\operatorname{CON}(G, k), u$ is connected to $v$ in path $p$ with the removal of any $k-1$ vertices in $G$. If $p \subseteq E-E_{s}, u$ is $k$-connected to $v$ in $G^{\prime \prime}$. Otherwise, $\exists\left(a_{1}, a_{2} \in p\right) \wedge\left(a_{1}, a_{2} \in V_{s}\right), u$ is connected to $a_{1}$ and $a_{2}$ is connected to $v$ in $G-G_{s}$. Since $\operatorname{CON}\left(G_{s}^{\prime}, k\right), a_{1}$ is connected to $a_{2}$ by removing those $k-1$ vertices. Then $u$ is connected to $v$ with the removal of any $k-1$ vertices in $G^{\prime \prime}$.

Table 1 Average message complexity in a sub-AS

\begin{tabular}{c}
\hline steps in each phase \\
All border nodes report their border lists to the parent core. \\
Core node distribute $D_{I A}$ vector to its border nodes. \\
Border nodes send $D_{I A}$ vector to border nodes of other sub-AS. \\
Border nodes of other sub-AS report $D_{I A}$ vector to their parent core. \\
Core node sends the link list to the sub-AS members.
\end{tabular}


Corollary 2 Let $G_{1}, G_{2}, \cdots, G_{n}$ be $k$-connected subgraphs of $k$-connected graph $G$. Let $G_{1}{ }^{\prime}, G_{2}{ }^{\prime}, \cdots, G_{n}{ }^{\prime}$ be edges reduction of $G_{1}, G_{2}, \cdots, G_{n}$, and $G_{1}{ }^{\prime}, G_{2}{ }^{\prime}, \cdots, G_{n}{ }^{\prime}$ are $k$-connected. Then, $G^{\prime \prime}=\left(G-\bigcup_{i=1}^{n} G_{i}\right) \bigcup_{G}\left(\bigcup_{i=1}^{n} G_{i}^{\prime}\right)$ is $k$-connected.

Lemma 3 Let $G=(V, E)$ be the initial topology of an AS. Let $G^{\prime}=\left(V, E^{\prime}\right)$ be the topology after Algorithm 1 is completed. Let $G_{i}=\left(V_{i}, E_{i}\right)$ be sub-AS networks, where $i=1, \cdots, n$. Let $G_{i}{ }^{\prime}=\left(V_{i}, E_{i}{ }^{\prime}\right)$, where $E_{i}{ }^{\prime}=E_{i}$ $\cap E^{\prime}$. Then, $\forall i, j$ subject to $1 \leqslant i \leqslant j \leqslant n$, we have $\operatorname{MCON}_{G}\left(G_{i}, G_{j}, k\right) \Rightarrow \operatorname{MCON}_{G^{\prime}}\left(G_{i}{ }^{\prime}, G_{j}{ }^{\prime}, k\right)$.

Proof of Lemma 3 Since nodes of any intra-sub-AS are $k$-connected. We take a sub-AS as a node here. Formally, we represent graph $G$ as $\bar{G}=\left(V_{S}, E_{S}\right)$, where $V_{S}=\left\{G_{1}, G_{2}, \cdots, G_{n}\right\}$ and $E_{S}=\left\{\left(G_{i}, G_{j}\right) \mid N B R_{G}\left(G_{i}, G_{j}\right.\right.$, $k)\}$. Actually, edge $\left(G_{i}, G_{j}\right)$ contains at least $k$ disjoint paths between $G_{i}$ and $G_{j}$. Let $\bar{G}^{\prime}=\left(V_{S}, E_{S}{ }^{\prime}\right)$ be the subAS level representation of $G^{\prime}$, where $E_{S}{ }^{\prime}=\left\{\left(G_{i}{ }^{\prime}, G_{j}{ }^{\prime}\right)\right.$ $\left.\mid N B R_{G^{\prime}}\left(G_{i}{ }^{\prime}, G_{j}{ }^{\prime}, k\right)\right\}$. We use $V_{S}$ to represent the set of sub-AS networks in $\bar{G}^{\prime}$, because we need not to consider the topology of intra-sub-AS (both $G_{i}$ and $G_{i}{ }^{\prime}$ are $k$-connected). We take all of them as nodes, so we consider $\left(G_{i}, G_{j}\right)$ and $\left(G_{i}^{\prime}, G_{j}^{\prime}\right)$ as the same edge. Recall that in Algorithm 1, each edge $\left(G_{i}, G_{j}\right) \in E_{S}$ has a weight $D_{I A}\left(G_{i}, G_{j}\right)$.

In order to prove Lemma 5, it suffices to show that $\forall G_{i}, G_{j} \in \bar{G}, G_{i}$ is connected to $G_{j}$ in $\bar{G}^{\prime}$. We order all edges in $\bar{G}$ in the ascending sequence of weights, and then judge whether an edge should be place into $\bar{G}^{\prime}$. Without loss of generality, let the ordering be $\left(e_{1}\right.$, $\left.e_{2}, \cdots, e_{m}\right)$, where $m=\left|E_{S}\right|$. Then we prove Lemma 5 by induction.

Base: Obviously, the pair of sub-AS networks corresponding to edge $e_{1}$ should always be placed into $\bar{G}^{\prime}$, i.e., $e_{1} \in E_{S}{ }^{\prime}$.

Induction: $\forall t \leqslant m$, if for all $q<t$, the pair of subAS networks corresponding to $e_{q}$ are connected in $\bar{G}^{\prime}$ (either directly or indirectly). And suppose $e_{t}=\left(G_{i}\right.$,
$\left.G_{j}\right)$. From Algorithm 1, the only reason why $e_{t} \notin E_{S}{ }^{\prime}\left(G_{i}\right.$ is not directly connected to $G_{j}$ in $\left.\bar{G}^{\prime}\right)$ is that there exists another sub-AS $G_{l}$, where both $D_{I A}\left(G_{i}, G_{l}\right)$ and $D_{I A}\left(G_{l}\right.$, $\left.G_{j}\right)$ is less than $D_{I A}\left(G_{i}, G_{j}\right)$. However, since edges $\left(G_{i}\right.$, $\left.G_{l}\right)$ and $\left(G_{l}, G_{j}\right)$ come before $\left(G_{i}, G_{j}\right)$ in the ascending order. From path $\overline{G_{i} G_{l} G_{i}}, G_{i}$ is connected to $G_{j}$ in $\bar{G}^{\prime}$.

By induction, we prove that $G_{i}$ is connected to $G_{j}$ in $\bar{G}^{\prime}$, and then $\operatorname{MCON}_{G}\left(G_{i}, G_{j}, k\right) \Rightarrow \operatorname{MCON}_{G^{\prime}}\left(G_{i}^{\prime}\right.$, $\left.G_{j}^{\prime}, k\right)$.

Finally, we prove the correctness of Theorem 1. In the proof, $G_{i}$ and $G_{i}^{\prime}$ have the same definition in Lemma 3.

Proof of Theorem 1 For every sub-AS $G_{i}$, we know that $\operatorname{CON}\left(G_{i}, k\right)$ is true. Then we partition those sub-AS networks into sets $A_{1}, \cdots, A_{s}$, where each set contains sub-AS networks are multihop $k$-connected in $G$, i.e., $\forall r=1, \cdots, s$, then, $\left(G_{i} \in A_{r}\right) \wedge\left(M C O N_{G}\right.$ $\left.\left(G_{i}, G_{j}, k\right)\right) \Rightarrow G_{j} \in A_{r}$. Then we define sets $A_{1}{ }^{\prime}, \cdots, A_{s}{ }^{\prime}$, where $\forall i, G_{i} \in A_{r} \Rightarrow G_{i}^{\prime} \in A_{r}{ }^{\prime}$. Applying Lemma 3 here, for every $A_{r}{ }^{\prime}=\left\{G_{r_{1}}{ }^{\prime}, \cdots, G_{r_{m}}{ }^{\prime}\right\}, \forall 1 \leqslant i<$ $j \leqslant m$, we have $\operatorname{MCON}_{G^{\prime}}\left(G_{r_{i}}{ }^{\prime}, G_{r_{j}}{ }^{\prime}, k\right)$. Take $A_{r}{ }^{\prime}$ as a sub-graph of $G^{\prime}, A_{r}^{\prime}=\left(V_{A_{r}{ }^{\prime}}, E_{A_{r}}\right)$, where $V_{A_{r}^{\prime}}=\{v \mid v \in$ $\left.A_{r}{ }^{\prime}\right\}$ and $E_{A_{r}^{\prime}}=\left\{(u, v) \mid\left(u, v \in A_{r}{ }^{\prime}\right) \wedge\left((u, v) \in E^{\prime}\right)\right\}$. Since $A_{r}^{\prime}$ only contains multihop $k$-connected subgraphs, applying Corollary 1 here, we have $A_{r}{ }^{\prime}$ is $k$-connected. Then, applying Corollary 2 here, we have $G^{\prime}=\left(G-\left(\bigcup_{r=1}^{s} A_{r}\right)\right) \bigcup_{G}\left(\bigcup_{r=1}^{s} A_{r}^{\prime}\right)$ is $k$-connected. Then $\operatorname{CON}(G, k) \Leftrightarrow \operatorname{CON}\left(G^{\prime}, k\right)$.

\section{Simulation results and discussions}

In this section, we present a set of simulation results to evaluate the effectiveness of the proposed intersub-AS topology control algorithm. These simulations were carried out using the NS2 simulator.

In the simulation study, nodes are randomly distributed in a $2000 \times 2000 \mathrm{~km}^{2}$ region. In order to study the effect of sub-AS size on the resulting topologies, we vary the number of nodes in the 
region among $125,150,175,200,225,250$. The maximal transmission range $R_{\max }$ of each node is $350 \mathrm{~km}$.

For each AS network, we consider:

1) $k$-connectivity: $k=1$ and $k=2$;

2) algorithms: Firstly, nodes in the AS network autonomously form sub-AS networks and elect sub-AS cores using VDBP ${ }^{[26]}$. Secondly, the proposed inter-sub-AS topology control algorithm is executed.

3) 1000 Monte Carlo simulations.

We configure that each node is at most one hop away from its parent core. In our simulations, the VDBP algorithm generates sub-AS networks where the average number of nodes per sub-AS is 6.7, 7.7, 8.6, 9.7 and 10.2 (results of 1000 simulations), respectively.

In Fig.7, we show average and maximum delay between two sub-AS networks which obtained from the proposed algorithm. Note that, we only consider link propagation delay in the simulation. It is evident from those results that the proposed algorithm is effective in reducing the delay between nodes. Recall that the maximal transmission range $R_{\max }$ of one node is $350 \mathrm{~km}$. The corresponding delay is 1.167 ms. When $k=1$, the proposed algorithm reduces the maximum delay to $0.864 \mathrm{~ms}$ when there are 125 nodes in the AS and as low as $0.577 \mathrm{~ms}$ when there are 225 nodes. For the average delay, the proposed algorithm reduces the delay to $0.524 \mathrm{~ms}$ when there are 125 nodes in the AS and as low as $0.354 \mathrm{~ms}$ when there are 225 nodes. When $k=2$, both the maximum and average delay results are higher than when $k=$ 1. That is expected because 2-connected is a stronger property than 1-connected. This is the consequence of having to maintain another higher delay link between adjacent sub-AS networks.

Fig.8 illustrates the number of messages exchanges required per node to complete the proposed algorithm in our simulation environment. Recall that the message complexity of the proposed algorithm is $o(N)+o\left(S_{N} S\right)$. For each node, the average number of

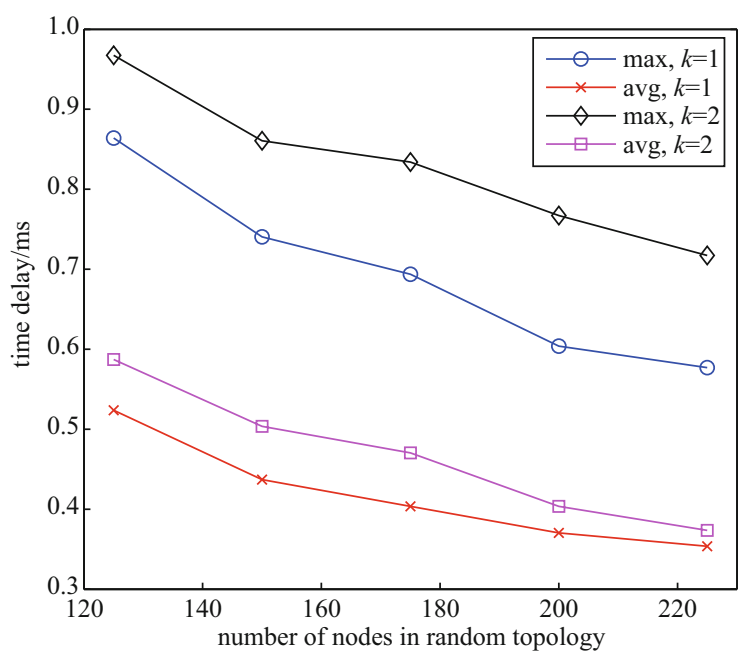

Figure 7 Delay results from the proposed topology control algorithm when $k=1$ and $k=2$

messages required is $\left(o(N)+o\left(S_{N} S\right)\right) / N=o(1)$. The result validates the analysis. When number of nodes in the AS increases from 125 to 225 , the average number of messages required per node does not increase. This shows the proposed algorithm has little extra overhead.

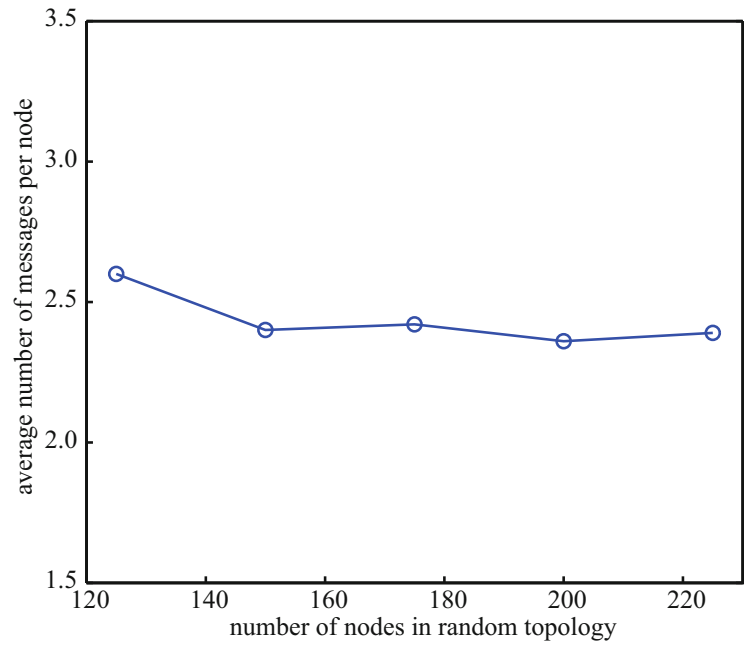

Figure 8 Number of messages exchanges per node when the number of nodes in the AS increases

\section{Conclusion}

In this study, we investigated the SIN architecture and topology control. We first proposed a novel 
SIN architecture, which was composed of GEO satellites as backbone network nodes, LEO or other satellite types as enhanced coverage nodes, and highaltitude platforms to meet the service requirements of emergency or hot-spot applications. Then, we proposed a hierarchical AS network model, which decoupled the complex SIN into simple sub-AS networks, and made the SIN's topology control easier to carry out. Finally, we proposed a topology control algorithm to minimize the time delay among subAS networks. We proved that the proposed algorithm achieved logical $k$-connectivity on the condition that the original physical topology had $k$-connectivity. Our simulation results validated the theoretic analysis and effectiveness of the proposed algorithm.

Although the assumptions stated in Section 3 are widely used in existing topology algorithms, some of them may not be practical. Our future work will focus on relaxing these constraints (e.g., nodes in one AS are homogeneous) for the proposed algorithm so as to improve its practicality in real applications. Furthermore, we will also consider the topology control of AS-1 (composed of satellites) in the near future.

\section{References}

[1] WANG J L, LIU C S. Development and application of INMARSAT satellite communication system[C]/Proceedings of the IEEE International Conference on Control, 2011: 619-621.

[2] BLUMENTHAL S H. Medium Earth orbit Ka band satellite communication system[C]//Proceedings of the IEEE Military Communications Conference, 2013: 273-277.

[3] CECCHINI J. Next generation GPS-aided space navigation system[J]. IEEE aerospace and electronic systems magazine, 2002, 17(12): 7-10.

[4] NOSCHESE P, PORFILI S, DI GIROLAMO S. ADS-B via iridium NEXT satellites[C]//Proceedings of the Digital Communications Conference, 2011: 213-218.

[5] PREVOT T. NextGen technologies for mid-term and far-term air traffic control operations $[\mathrm{C}] /$ Proceedings of IEEE Digital Avionics Systems Conference, 2009: 1-16.

[6] MUKHERJEE J, RAMAMURTHY B. Communication technologies and architectures for space network and interplanetary internet[J]. IEEE communication surveys and tutorials, 2013, 15(2): 881-897.
[7] BHASIN K B, HAYDEN J K. Architecting communication network of networks for space system of systems[C]/Proceedings of the IEEE System of Systems Engineering Conference, 2008: 1-7.

[8] HU H F, LIU Y A. A feasible mesh-based architecture and protocol model of space information network[C]/Proceedings of the IEEE Geoscience and Remote Sensing Conference, 2010: 529-531.

[9] REN F, FAN J L. An adaptive distributed certificate management scheme for space information network[J]. IET information security, 2013, 7(4): 318-326.

[10] ZHANG G X, ZHANG W, ZHANG H, et al. A novel proposal of architecture and network model for space communication networks[C]/Proceedings of the IAF 65th International Astronautical Congress, 2014: 1-7.

[11] NISHIYAMA H, TADA Y, KATO N, et al. Toward optimized traffic distribution for efficient network capacity utilization in two-layered satellite networks[J]. IEEE transactions on vehicular technology, 2013, 62(3): 1303-1313.

[12] LI Y J, ZHAO S H, WU J L, et al. Designing of a novel optical two layered satellite network[C]/Proceedings of IEEE Computer Science and Software Engineering Conference, 2008: 976-979.

[13] YIN Z Z, ZHANG L, ZHOU X W, et al. Qos-aware multicast routing protocol for triple-layered $\mathrm{LEO} / \mathrm{HEO} / \mathrm{GEO}$ satellite IP network[C]//Proceedings of IEEE Global Mobile Congress,2010: 1-6.

[14] TALEB T, FADLULLAH Z M , TAKAHASHI T, et al. Tailoring ELB for multi-layered satellite network[C]//Proceedings of IEEE Communications Conference, 2009: 1-5.

[15] WANG J F, HU Y M, ZHOU H X, et al. Topological dynamics characterization for layered satellite networks[C]/Proceedings of IEEE Performance Computing and Communication Conference, 2006: 349-356.

[16] KIMURA K, INAGAKI K, KARASAWA Y. Double-layered inclined orbit constellation for advanced satellite communication network[J]. IEICE transactions on communication, 1997, 1(1): 93-102.

[17] LEE J, KANG S. Satellite over satellite (SoS) metwork: a novel architecture for satellite network[C]//Proceedings of IEEE INFOCOM, 2000: 315-321.

[18] AKYILDIZ I F, EKICI E, BENDER M D. MLSR: a novel routing algorithm for multi-layered satellite IP networks[J]. IEICE transactions on networking, 2002, 10(3): 411-424.

[19] GRAZIANO M D. Overview of distributed missions[J]. Distributed space missions for Earth system monitoring, 2013, 31(1): 375-386.

[20] CORNFORD S, SHISHKO R, WALL S, et al. Evaluating a fractionated spacecraft system: a business case tool for DARPA's F6 program[C]//Proceedings of IEEE Aerospace Conference, 2012: $1-20$.

[21] MOHAMMED A, MEHMOOD A, PAVLIDOU F N, et al. The role of high altitude platforms in the global wireless connectivity[J]. Proceedings of the IEEE, 2011, 99(11): 1939-1953.

[22] AVAGNINA D, DOVIS F, GHIGLIONE A, et al. Wireless networks based on high altitude platforms for the provision of integrated navigation/communication services[J]. IEEE communications magazine,2002, 40(2): 119-125. 
[23] LIU J, LIN C, YE N, et al. Importance evaluation algorithm of links and nodes in space information networks[C]/Proceedings of the IEEE Internet Technology and Applications Conference, 2011: 1-4.

[24] YE N, ZHU Z L, SHI J P. Distributed cluster-based fault-tolerant topology control for space information networks[C]//Proceedings of IEEE Cyber-Enabled Distributed Computing and Knowledge Discovery Conference,2010: 210-217.

[25] JAIKAEO C, SHEN C C. Adaptive backbone-based multicast for
Ad hoc networks[C]/Proceedings of IEEE International Conference on Communications, 2002: 3149-3155.

[26] AZAD A, HALAPPANAVAR M, RAJAMANICKAM S, et al. Multi-threaded algorithms for maximum matching in bipartite graphs[C]//Proceedings of IEEE Parallel and Distributed Processing Symposium, 2012: 860-872.

[27] BONDY J A, MURTY U S R. Graph theory[M]. Berlin: Springer, 2008.

\section{About the authors}

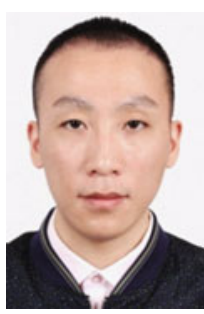

ZHANG wei [corresponding author] received the B.S. degree (with honors) in communication engineering from Xidian University, Xi'an, China, in 2009, and the M.S. and Ph.D. degrees from College of Communication Engineering, PLA University of Science and Technology, Nanjing, China, in 2012 and 2016, respectively. In 2016, he joined the Department of Optical and Electrical Equipment, Equipment Academy, Beijing, where he is currently a lecturer. His research interests include signal processing in TT\&C system and space information networks. (Email: zwv@163.com)

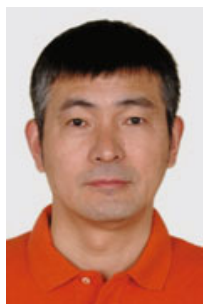

ZHANG Gengxin received his M.S. and Ph.D. degrees from the Institute of Communication Engineer, Nanjing, China in 1990 and 1993 respectively. $\mathrm{He}$ is currently a Professor in PLAUST (PLA University of Science and Technology), Nanjing, China. His research interests include the design of communication systems, satellite and deep space communications. (Email: satlab@126.com)

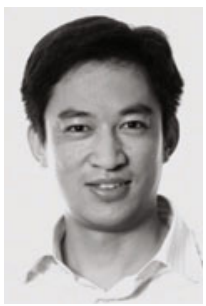

XIE Zhidong received his Ph.D. degree from PLAUST (PLA University of Science and Technology), Nanjing, China in 2012. He is currently a lecturer in PLAUST. His research interests mainly fall in the area of wireless communications, satellite communications and deep space communications. (Email: xzd313@163.com)

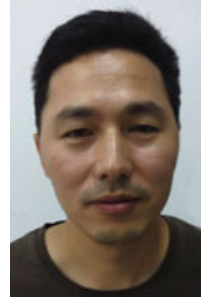

BIAN Dongming received his $\mathrm{Ph} . \mathrm{D}$. degree from PLAUST (PLA University of Science and Technology), Nanjing, China, in 2004. He is currently a professor in PLAUST. His research interests mainly fall in the area of wireless communications, satellite communications and deep space communications. (Email: bian_dm@163.com)

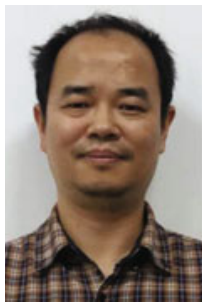

LI Yongqiang received his Ph.D. degree from PLAUST (PLA University of Science and Technology), Nanjing, China, in 2009. He is currently a lecturer in PLAUST. His research interests mainly fall in the area of wireless communications, satellite communications and deep space communications. (Email: niceme@ vip.sina.com) 